\title{
Air-to-Air Missile Tracking and Guidance Law Identification Based on CKF
}

\author{
Wu Xiao-tian, Yang Jin-heng, Wang Xing, Zhang Xiang \\ Aeronautics and Astronautics Engineering College \\ Air Force Engineering University,Xi’an, China \\ celeswu@sina.com,kellyyoung331@gmail.com,13399289501@189.cn,cmaristotle@163.com
}

Keywords: guidane law identification; cubature Kalman filter; augmented proportional navigation; missile tracking

\begin{abstract}
An air-to-air missile tracking and guidance law identification method based on CKF is proposed. A precise air-to-air missile model is constructed with drag force taken into consideration. With CKF method and preprocessing, guidance law filter is designed for missile tracking and estimation of guidance law, gravity compensation and drag constant. Simulation results indicate the rapidness and accuracy of this method.
\end{abstract}

\section{Introduction}

Aircrafts are with high combat values in the battlefield, which makes it very important to research methods to enhance survivability of aircrafts. With development of air-to-air missiles, effectiveness of traditional countermeasures like active and passive jamming is increasingly limited. Novel countermeasure method is one heated research topic around the world. One key technique of novel countermeasure is air-to-air missile tracking and guidance law identification.

Whatever design of evasive guidance law of aircrafts or guidance law of defender missiles, model of air-to-air missile is needed [1]. Furthermore, air-to-air missile tracking brings missile trajectory and time-to-go estimation [2], which are of great benefits for pilots to make decisions.

There are few literatures on the topic of air-to-air missile tracking and guidance law identification.

Guidance law identification of ground-to-air missile is studied in [2] through maximum likelihood method; possibilities of different guidance laws are determined using Bayesian reasoning in [3]; guidance law identification is achieved through MMAE and IMM method in [1] and [4], respectively; research is extended in three-dimensional coordinates in [5] and saturation condition of missile controller is taken into account in [6].

Some rational assumptions are included in these papers to simplify model while limit range of applicability at the same time. The opposability of air-to-air missiles and aircrafts is with such natural characteristics like high speed, wide region and high maneuvering. The above literatures base on ideal condition and overlook the drag force, they are not adaptable for guidance law identification of air-to-air missile and long-time tracking, which relay on more realistic air-to-air missile models.

In the beginning, an air-to-air missile model of three-degree of freedom is set up in this paper. Then a guidance law filter is designed with missile model, CKF (Cubature Kalman Filter) method and preprocessing. Compared with traditional literatures, the use of CKF can provide higher precision and less computational load. In this paper, APN (Augmented Proportional Navigation) is the research object. APN is close to the optimal guidance law in form and is prone to realize in engineering $[7,8]$, it is widely used in air-to-air missile. Furthermore, other guidance law mentioned in [1] can be approximated by APN with different navigation constants [6].

\section{Model of Air-to-Air Missile}

Geometry relationship between the air-to-air missile (known as missile later) and the evading aircraft (known as the target later) is shown in figure 1. 


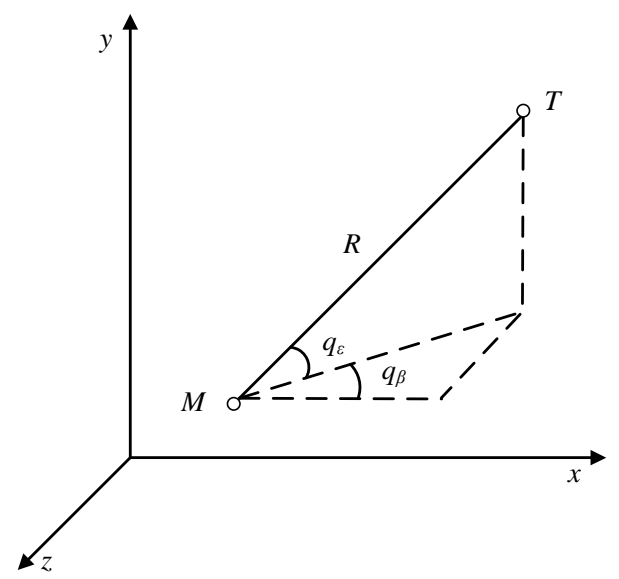

Figure 1 Geometry relationship

$q_{\varepsilon}$ and $q_{\beta}$ are lines of sight from the missile to the plane and $R$ refers to the distance, they are described by the following equation $[9,10]$ :

$$
\left\{\begin{array}{l}
R^{2}=x_{r}^{2}+y_{r}^{2}+z_{r}^{2} \\
q_{\varepsilon}=\arcsin \left(\frac{z_{r}}{R}\right) \\
q_{\beta}=\arctan \left(\frac{z_{r}}{x_{r}}\right)
\end{array}\right.
$$

where $x_{r}, y_{r}, z_{r}$ is distance between the missile and the target in $x, y, z$ axis, respectively. The differential form of equation (1) is

$$
\left\{\begin{array}{l}
d R=\frac{x_{r} d x_{r}}{R}+\frac{y_{r} d y_{r}}{R}+\frac{z_{r} d z_{r}}{R} \\
d q_{\varepsilon}=\frac{R d y_{r}-y_{r} d R}{R \sqrt{x_{r}^{2}+z_{r}^{2}}} \\
d q_{\beta}=\frac{x_{r} d z_{r}-z_{r} d x_{r}}{x_{r}^{2}+z_{r}^{2}}
\end{array}\right.
$$

The kinetic equation of the missile in inertial coordinate is

$$
\left\{\begin{array}{l}
\dot{x}_{m}=v_{m} \cos \theta \cos \varphi_{V} \\
\dot{y}_{m}=v_{m} \sin \theta \\
\dot{z}_{m}=-v_{m} \cos \theta \sin \varphi_{V}
\end{array}\right.
$$

where $x_{m}, y_{m}$ and $z_{m}$ refer to position of the missile in inertial coordinate, the remaining three variables are the flight path angle $\theta$, the heading angle $\varphi_{V}$, and the velocity $v_{m}$. The kinematic equation of three-degree of freedom is

$$
\left\{\begin{array}{l}
\dot{\theta}=\left(n_{y}-g \cdot \cos \theta\right) / v_{m} \\
\dot{\varphi}_{V}=-n_{z} / v_{m} \cos \theta \\
\dot{v}_{m}=\frac{1}{m(t)}[T(t)-D]-g \sin \theta
\end{array}\right.
$$

where $n_{y}$ and $n_{z}$ is pitch and yaw control overload of the missile, respectively. They are determined by guidance law. $m(t)$ denotes mass of the missile, $T(t)$ the thrust force, $D$ the drag force. The major part of missile flight is the passive flying stage, when engine is turning off, which means $T(t)=0$. The drag force is given by

$$
D=\frac{1}{2} \rho v_{m}^{2} S_{m} C_{m}
$$

where the air density $\rho$ is taken from the International Standard Atmosphere, $S_{m}$ is the reference wing 
area and $C_{m}$ the drag coefficient, which is determined by Mach number and attack angle. Although $C_{m}$ can be given by fitting relationship, it is a constant in simulation, considering the change of its value is very limited.

Control overload of APN is

$$
\left\{\begin{array}{l}
n_{y}=v_{r} \cdot N \cdot \dot{q}_{\varepsilon}+\frac{N}{2} \cdot n_{T y}+\alpha \cdot g \\
n_{z}=v_{r} \cdot N \cdot \dot{q}_{\beta}+\frac{N}{2} \cdot n_{T z}
\end{array}\right.
$$

where $n_{T y}$ and $n_{T z}$ refer to pitch and yaw overload of the target, $N$ denotes navigation constant, $\alpha$ is added for the compensation of gravity, and $v_{r}=\mathrm{d} R / \mathrm{d} t$.

For the purpose of missile tracking and guidance law identification, the state vector is determined as

$X=\left[x_{m}, y_{m}, z_{m}, v_{m}, \theta, \varphi_{V}, N, \alpha, k\right]^{T}$, where $k$ is known as the drag force constant and $k=\frac{S_{m} C_{m}}{2 m}$.

Model of the missile is

$$
\left\{\begin{array}{l}
\dot{x}_{m}=v_{m} \cos \theta \cos \varphi_{V} \\
\dot{y}_{m}=v_{m} \sin \theta \\
\dot{z}_{m}=-v_{m} \cos \theta \sin \varphi_{V} \\
\dot{v}_{m}=-k \rho v_{m}{ }^{2}-g \sin \theta \\
\dot{\theta}=\left(n_{y}-g \cdot \cos \theta\right) / v_{m} \\
\dot{\varphi}_{V}=-n_{z} g / v_{m} \cos \theta \\
\dot{N}=0 \\
\dot{\alpha}=0 \\
\dot{k}=0
\end{array}\right.
$$

The target can obtain information of its own position through GPS or inertial navigation system. And under the assumption that airborne sensors can provide distances between the target and the missile in the three axis directions, the position of missile can be obtained. Hence, the measurement equation is

$$
Z=\left[x_{m}, y_{m}, z_{m}\right]^{T}
$$

\section{Guidance Law Identification Filter}

Guidance law identification includes multi variables, it is a high order nonlinear issue while CKF is one preferable algorithm for it at present. Similar to UKF (Unscented Kalman Filter), one order and two order moments needed in filtering are obtained through nonlinear calculation of sampling points with weights, which avoids linearization process of nonlinear issue. Furthermore, CKF overcomes the shortcoming of UKF that there appear sigma points of negative value in high order filtering, therefore, CKF is better in stability and accuracy [11]. The process of guidance law identification filter is as follows:

\subsection{Initialization}

Determine initial values of $\hat{X}_{0}, P_{0}, Q_{0}$ and $R_{0}$.

The state vector includes the position, velocity, flight path and heading angle of the missile, navigation, gravity compensation and drag constant. Among them, position is initialized by observation and constants are set among rational range. As for velocity, flight path and heading angle, a preprocessing method is proposed for their initialization. Equation (3) can be transformed as the following equation: 


$$
\left\{\begin{array}{l}
v_{m}=\sqrt{\dot{x}_{m}^{2}+\dot{y}_{m}^{2}+\dot{z}_{m}^{2}} \\
\theta=\sin ^{-1}\left(\sqrt{\frac{\dot{y}_{m}^{2}}{\dot{x}_{m}^{2}+\dot{z}_{m}^{2}}}\right) \\
\varphi_{V}=\tan ^{-1}\left(-\frac{\dot{z}_{m}}{\dot{x}_{m}}\right)
\end{array}\right.
$$

Equation (9) show velocity, flight path and heading angle can be calculated with velocities in three coordinates' directions, which root in observation data. During preprocessing, velocities in three coordinates' directions are considered constant, and n sets of observation data are obtained as $\left[Z_{1}, Z_{2}, \cdots Z_{n}\right]$. As observation error is Gaussian distribution, observation data are processed using least square method:

$$
\left\{\begin{array}{c}
\hat{\dot{x}}_{m}=\frac{\sum_{i=1}^{n} t_{i} Z_{i}(1)-n \bar{t} \cdot \bar{Z}(1)}{\sum_{i=1}^{n} t_{i}^{2}-n \bar{t}^{2}} \\
\hat{\dot{y}}_{m}=\frac{\sum_{i=1}^{n} t_{i} Z_{i}(2)-n \bar{t} \cdot \bar{Z}(2)}{\sum_{i=1}^{n} t_{i}^{2}-n \bar{t}^{2}} \\
\hat{\dot{Z}}_{m}=\frac{\sum_{i=1}^{n} t_{i} Z_{i}(3)-n \bar{t} \cdot \bar{Z}(3)}{\sum_{i=1}^{n} t_{i}^{2}-n \bar{t}^{2}}
\end{array}\right.
$$

where $t_{i}=i \times \Delta t, \Delta t$ is the observation interval. Initial value of velocity, flight path and heading angle are determined applying the result of equation (10) in equation (9).

\section{A. Prediction}

$$
\begin{aligned}
& S(k-1 \mid k-1)=\sqrt{P(k-1 \mid k-1)} \\
& \mathcal{X}_{i}(k-1 \mid k-1)=\hat{X}(k-1 \mid k-1)+S(k-1 \mid k-1) \xi_{i}
\end{aligned}
$$

where

$$
\xi_{i}=\sqrt{\frac{m}{2}}[1]_{i} \quad m=2 n
$$

[1] denotes point set of $n$-dimension space.

$$
\begin{aligned}
\hat{X}_{i}(k \mid k-1)= & f\left[X_{i}(k-1 \mid k-1)\right] \\
\hat{X}(k \mid k-1)=\frac{1}{m} \sum_{i=1}^{m} \hat{X}_{i}(k \mid k-1) & \\
P(k \mid k-1)= & \frac{1}{m} \sum_{i=1}^{m}\left[\hat{X}_{i}(k \mid k-1)-\hat{X}(k \mid k-1)\right] . \\
& {\left[\hat{X}_{i}(k \mid k-1)-\hat{X}(k \mid k-1)\right]^{T}+Q_{k-1} }
\end{aligned}
$$

\subsection{Update}

$$
\begin{aligned}
& S(k \mid k-1)=\sqrt{P(k \mid k-1)} \\
& \mathcal{X}_{i}(k \mid k-1)=\hat{X}(k \mid k-1)+S(k \mid k-1) \xi_{i} \\
& \hat{Z}_{i}(k \mid k-1)=h\left[X_{i}(k \mid k-1)\right]
\end{aligned}
$$




$$
\begin{gathered}
\hat{Z}(k \mid k-1)=\frac{1}{m} \sum_{i=1}^{m} \hat{Z}_{i}(k \mid k-1) \\
S_{k}=\frac{1}{m} \sum_{i=1}^{m}\left[\hat{Z}_{i}(k \mid k-1)-\hat{Z}(k \mid k-1)\right] \\
\cdot\left[\hat{Z}_{i}(k \mid k-1)-\hat{Z}(k \mid k-1)\right]^{T}+R_{k} \\
C_{k}=\frac{1}{m} \sum_{i=1}^{m}\left[X_{i}(k \mid k-1)-\hat{X}(k \mid k-1)\right] \\
\cdot\left[\hat{Z}_{i}(k \mid k-1)-\hat{Z}(k \mid k-1)\right]^{T} \\
K_{k}=C_{k} S_{k}^{-1}
\end{gathered}
$$

The state estimation and covariance estimation are

$$
\begin{gathered}
\hat{X}(k \mid k)=\hat{X}(k \mid k-1)+K_{k}[Z(k)-\hat{Z}(k \mid k-1)] \\
P(k \mid k)=P(k \mid k-1)-K_{k} S_{k} K_{k}{ }^{T}
\end{gathered}
$$

\section{Numerical Simulation}

In this section, different cases are considered to illustrate the performance of guidance law estimation and missile tracking.

The missile is launched at $t_{0}=0 \mathrm{~s}$, with initial position $[0 \mathrm{~m}, 7000 \mathrm{~m}, 0 \mathrm{~m}]$, initial velocity $v_{m}=1.2 \mathrm{Ma}$, initial mass $m_{0}=200 \mathrm{~kg}$, initial flight path angle $\theta=0^{\circ}$, initial heading angle $\varphi_{V}=0^{\circ}$, navigation constant $N=4$, gravity compensation constant $\alpha=1$, wing area $S_{m}=0.3 \mathrm{~m}^{2}$, drag force coefficient $C_{m}=0.55$. Engine of the missile works for $10 \mathrm{~s}$, with $20 \mathrm{kN}$ thrust force and $7 \mathrm{~kg} / \mathrm{s}$ flow rate. It is assumed that the missile has perfect information of the target, which means LOS and maneuvering of the target are obtained without delay or error.

Target starts from $[10000 \mathrm{~m}, 9000 \mathrm{~m}, 10000 \mathrm{~m}]$, with initial flight path angle -pi/4, initial heading angle $0^{\circ}$, and constant velocity $v_{T}=0.8 \mathrm{Ma}$.

The sampling rate of the simulation is $0.01 \mathrm{~s}$, relative distance measurements are obtained by the target with a $50 \mathrm{~Hz}$ update rate when the distance between the target and the missile is less than $10 \mathrm{~km}$, and measurements are corrupted by independent zero-mean white Gaussian noise sequences with standard deviations of $20 \mathrm{~m}$.

\subsection{Simulation results for the target with no maneuvering}

In this case, control overload of the target stays zero all through simulation. Tracking and guidance law identification performance are shown in figure 2 and figure 3.

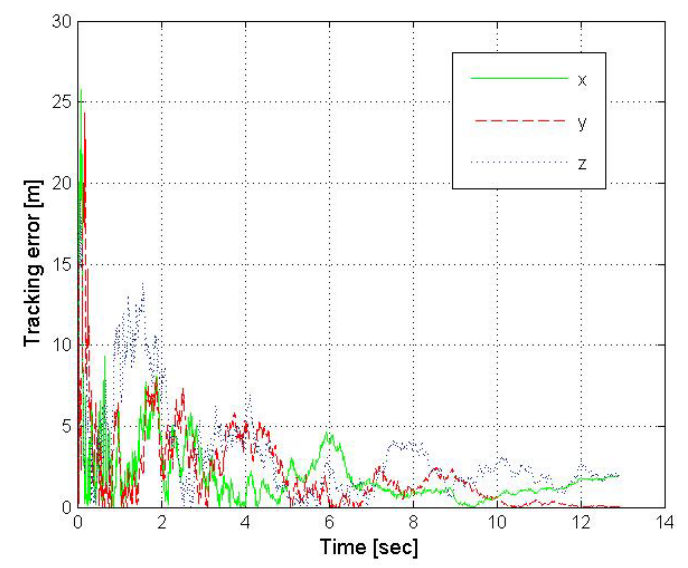

Figure 2 Tracking performance of case A 


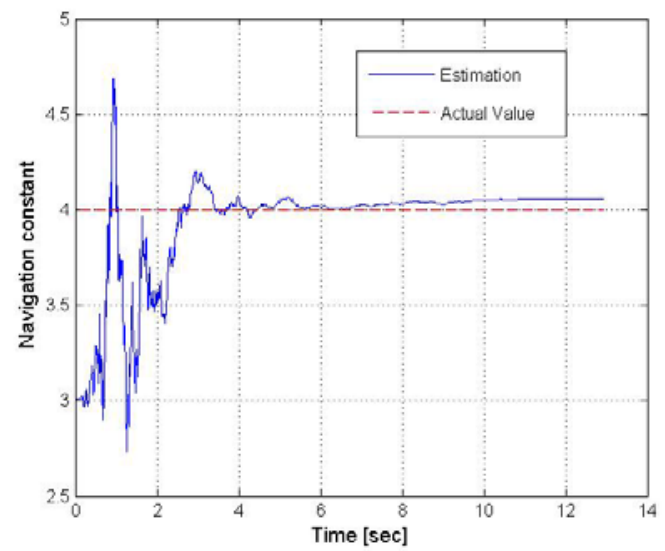

(a) Navigation constant estimation

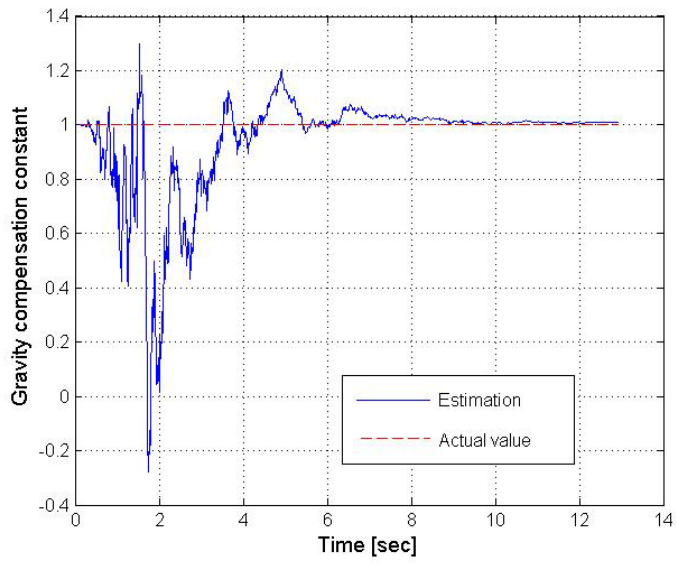

(b) Gravity compensation constant estimation

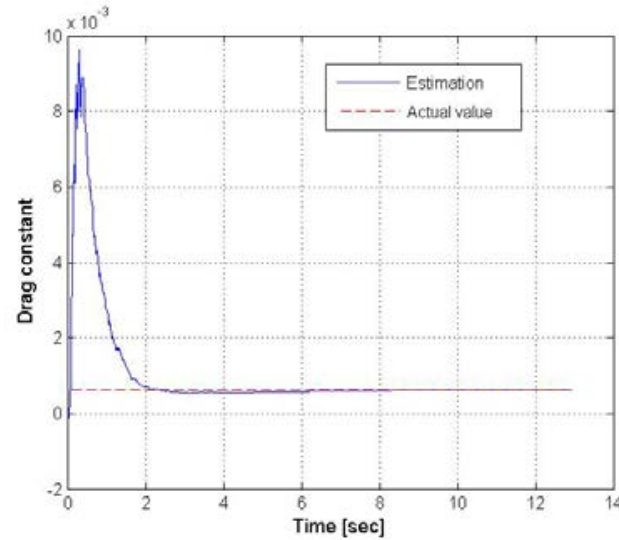

(c) Drag constant estimation

Figure 3 Guidance law identification performance

\subsection{Simulation results for the target with hover maneuvering}

This is the case in which the target start to conduct 8G hover maneuvering when the distance between the target and the missile is less than 8 kilometer. Tracking and guidance law identification performance are shown in figure 4 and figure 5 . 


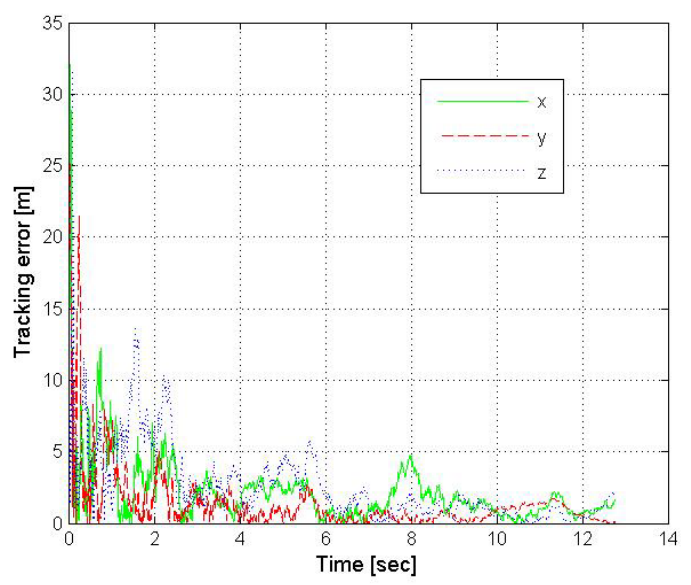

Figure 4 Tracking performance of case B
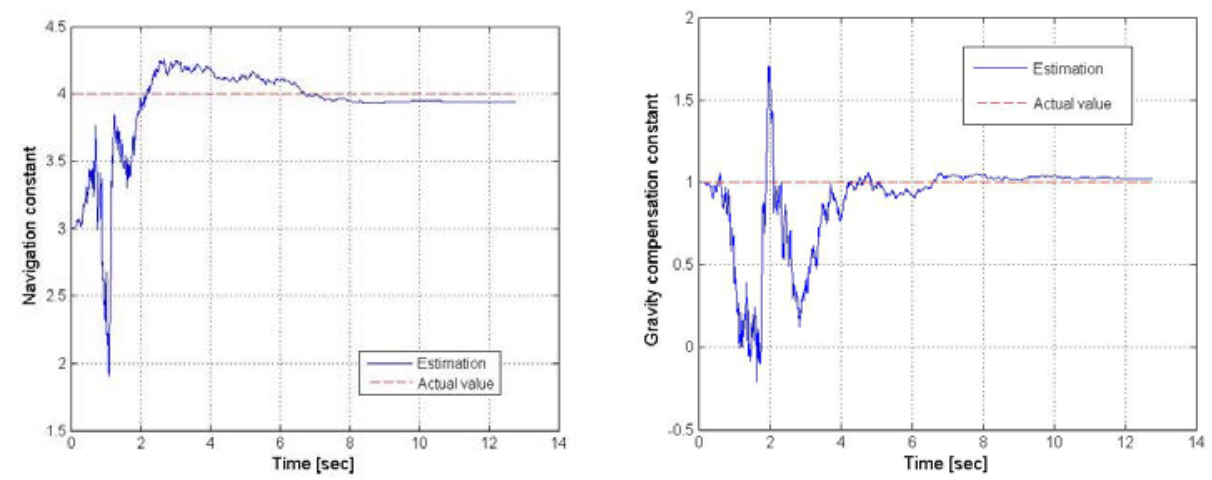

(a) Navigation constant estimation (b) Gravity compensation constant estimation

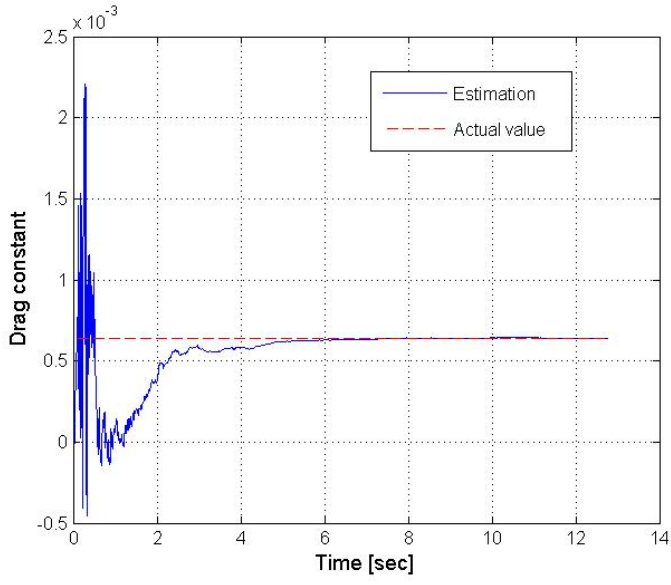

(c) Drag constant estimation

Figure 5 Guidance law identification performance

Results in figure 2 to figure 5 show the effectiveness of missile tracking method whatever maneuvering the target conducts. Estimations of all constants convergence to the actual value rapidly and stay stable in 4 to 6 s. The relative deviation is less than $5 \%$ for every constant.

Precise model and accurate estimation of constants guarantee missile tracking performance, tracking errors of missile position convergence to less than $5 \mathrm{~m}$ in $6 \mathrm{~s}$.

\section{Conclusion}

In this paper an air-to-air missile tracking and guidance law identification method based on airborne platform is presented. A more realistic air-to-air missile model is adapted taking the drag force into consideration. Combined with CKF method, the guidance law identification filter can provide accurate missile tracking and estimation of navigation, gravity compensation and drag 
constant. This method can also be used for the identification of other guidance laws or for the tracking of other kind of missiles as long as model is provided. The effectiveness for high order nonlinear issue of this method has been exhibited. It is practical for improving survivability of aircrafts.

\section{References}

[1] V. Shaferman and T. Shima, "Cooperative Multi-Model Adaptive Guidance for an Aircraft Defending Missile,” Journal of Guidance, Control and Dynamics, vol. 33, No.6, November. 2010, pp. 1801-1813.

[2] L. Lin, T. Kirubarajan and Y. Bar-Shalom, "Pursuer Identification and Time-To-Go Estimation using Passive Measurements from an Evader," IEEE Transactions on Aerospace and Electronic systems, vol. 41, January. 2005, pp.190-202.

[3] J. Karelahti and K. Virtanen, "Adaptive Controller for the Avoidance of an Unknownly Guided Air Combat Missile,” Proc. IEEE Conference on Decision and Control, IEEE Press, Dec.2007, pp. 1306-1313.

[4] J. Yun and C. Ryoo, "Missile Guidance Law Estimation Using Modified Interactive Multiple Model Filter,” Journal of Guidance, Control and Dynamics, vol. 37, March. 2014, pp. 484-496, doi:10.2514/1.61327.

[5] W. Xiao-ping, C. Yuan-li, Y. Zhen-hua, L. Qin-ying and Y. Xi-gui, "Nonlinear MMAE-based missile guidance law and parameter identification,” Flight Dynamics, vol. 33, No.5, October. 2015, pp. 430-434, doi:10.13645/j.cnki.f.d.20150702.004

[6] Z. Xing-guang, Z. Di, D. Run-le and L. Jia-qi, "PN Guidance Law Identification Using MultiModel Adaptive Estimation,” Journal of Astronautics, vol. 37, No.8, August. 2016, pp. 974-983

[7] Y. Yu and W. Yu-hang, “Acceleration Estimation of Maneuvering Targets Based on Extended State Observer," Systems Engineering and Electronics, vol. 31, No.11, November. 2009, pp. 2682-2692.

[8] R. Kumar and H. Seywald, "Near-Optimal Three-Dimensional Air-to-Air Missile Guidance Against Maneuvering Target,” Journal of Guidance, Control and Dynamics, vol. 18, No. 3, May. 1995, pp. 457-464.

[9] J. Karelahti, K. Virtanen, and T. Raivio, "Near-Optimal Missile Avoidance Trajectories via Receding Horizon Control,” Journal of Guidance, Control and Dynamics, vol. 30, September. 2007, pp. 1287-1298, doi:10.2514/1.26024.

[10] D. Xinghua, F. Yangwang, W. Youli and F. Xiaofei, "Simulation Analysis on air-to-air Missile Allowable Launch Envelope about Cooperative Air Combat of Multi-fighter Formation," Journal of Beijing University of Aeronautics and Astronautics, vol. 40, No.3, March. 2014, pp. 370-376

[11] I. Arasaratnam, S. Haykin and T. Hurd, "Cubature Kalman Filtering for ContinuousDiscrete Systems: Theory and Simulations,” IEEE Transactions on Signal Processing, vol. 58, No.10, October. 2010, pp. 1-16 